\title{
Experimental validation of additively manufactured optimized shapes for passive cooling
}

Lazarov, Boyan S.; Sigmund, Ole; Meyer, Knud E.; Alexandersen, Joe

Published in:

Applied Energy

Link to article, DOI:

10.1016/j.apenergy.2018.05.106

Publication date:

2018

Document Version

Early version, also known as pre-print

Link back to DTU Orbit

Citation (APA):

Lazarov, B. S., Sigmund, O., Meyer, K. E., \& Alexandersen, J. (2018). Experimental validation of additively manufactured optimized shapes for passive cooling. Applied Energy, 226, 330-339.

https://doi.org/10.1016/j.apenergy.2018.05.106

\section{General rights}

Copyright and moral rights for the publications made accessible in the public portal are retained by the authors and/or other copyright owners and it is a condition of accessing publications that users recognise and abide by the legal requirements associated with these rights.

- Users may download and print one copy of any publication from the public portal for the purpose of private study or research.

- You may not further distribute the material or use it for any profit-making activity or commercial gain

- You may freely distribute the URL identifying the publication in the public portal 


\title{
Optimized shapes for passive cooling
}

\author{
Boyan S. Lazarov ${ }^{\mathrm{a}, \mathrm{b}, 1}$, Ole Sigmund ${ }^{\mathrm{a}}$, Knud E. Meyer ${ }^{\mathrm{a}}$, Joe Alexandersen ${ }^{\mathrm{a}}$ \\ ${ }^{a}$ Department of Mechanical Engineering, Technical University of Denmark \\ Nils Koppels Allé, Building 404, Kgs. Lyngby, DK-2800, Denmark \\ ${ }^{b}$ School of Mechanical, Aerospace and Civil Engineering, \\ the University of Manchester, M13 9PL, Manchester, UK
}

\begin{abstract}
Lighting is an essential requisite for the industrial world but consumes vast amounts of energy. Hence, even minimal savings in energy consumption of lighting devices may have a significant effect on cost and environmental impact. Here we demonstrate a computer-aided approach for optimizing passive heat sinks for light-emitting diode (LED) lamps. Efficient passive cooling ensures lower energy consumption, increased lifetime and reduced maintenance costs of this rapidly growing, expectedly soon to be governing, lighting technology. Highly efficient cooling structures are generated by topology optimization, a computational morphogenesis approach with ultimate design freedom, relying on high-performance computing and simulation. The optimized devices, exhibiting complex and organiclooking topologies not unlike corals, are manufactured by additive manufacturing technology. Numerical simulations confirmed by experiments indicate that topology-optimized designs may outperform traditional pin-like geometries by more than $21 \%$, resulting in a doubling of life expectancy and $50 \%$ decrease in operational cost.
\end{abstract}

Keywords:

\section{Introduction}

Lighting in residential and commercial sectors accounts for up to $7 \%$ of the total US electricity consumption in $2016^{2}$ and accounts for a significant share $(14-60 \%)$ of the electricity bill in retail and office buildings $[1,2]$. Over the last decade, initiatives supported by governments around the world have accelerated development and adoption of light-emitting diode (LED) products as a replacement for century-old incandescent technologies and newer high-intensity discharge and fluorescent lamps. LED technologies are estimated to comprise anywhere from $25 \%$ to upwards of $80 \%$ of lighting by 2020, and their rapid development and improved efficiency will result in a $75 \%$ reduction in energy consumption for lighting by 2035 [2]. Other main positive factors are light output, longevity, light distribution, dimensions, chromaticity, control, stability, environmental impact, durability and cost.

Despite their efficiency compared to other lighting technologies, LEDs still only convert $25 \%$ to $35 \%$ of

\footnotetext{
${ }^{1}$ corresponding author: Boyan Lazarov (bslazarov@gmail.com)

${ }^{2}$ U.S. Energy Information Administration (EIA), www.eia.gov
}

the input energy to light, the rest is lost as heat generated in a relatively small area of the light-emitting semiconductor. This contrasts incandescent and fluorescent lamps, where heat generated in voluminous bulbs is easily conducted through bulb walls and subsequently radiated and convected through the surroundings. Increased temperature or overheating results in a shift of emission wavelength, lower lumen output and lifetime reduction of LEDs. Large LEDs produce more light per unit area and generate a significant amount of heat, thus requiring external heat exchangers which impacts size, reliability and cost. The excess heat must be removed either by active forced convection (fans) or by passive heat sinks relying on natural convection.

Fans are energy consuming, noisy and subject to mechanical failure, whereas natural convection needs careful design of heat sink geometries to ensure adequate cooling performance. A typical off-the-shelf fan, compatible with the dimensions of the presented coolers, consumes between $0.4 \mathrm{~W}$ and $1.2 \mathrm{~W}$, increasing the energy consumption with $8 \%$ to $24 \%$ compared to a passively cooled solution for a 5W LED illuminator.

Natural convection, on the other hand, is a heat transport mechanism which accelerates fluids based on dif- 
ferences in fluid densities due to temperature gradients. Figure 1 visualizes the process, where a sink attached to a heat source conducts heat to its surface, and the temperature difference between sink surface and ambient air generates a fluid motion which transfers the heat to the surroundings. The performance of the heat sink depends on a delicate balance between its ability to conduct heat away from the semiconductor and the excitation of a fluid motion strong enough to remove the heat by natural convection. The process continues to draw a lot of attention and has been studied extensively using experimental and numerical techniques, leading to a set of simple design rules utilized by designers and engineers. However, the simplicity of these rules, as well as the complexity of the physical phenomena may easily result in sub-optimal solutions leaving many opportunities for improvements $[3,4,5]$.

We use state-of-the-art computing techniques and algorithms to design efficient passive heat sinks and verify the resulting structures experimentally. We find that topology-optimized natural convection coolers outperform traditional designs. The computational morphogenesis approach results in coral-like branching topologies resembling plants and organisms found in nature. The complex topologies can be manufactured using additive manufacturing technologies, or may inspire simplified and more easily manufacturable designs with a minor loss in performance. The process is verified by numerical simulations and confirmed experimentally. Methodology and findings may be further utilized and extended to cooling of electronic equipment, industrial machines, and processes, where robustness, lowmaintenance cost and performance are of vital importance.

Figure 2a) shows a commercial LED bulb developed as a direct replacement for an incandescent one. Due to the small size of the semiconductor, designers have extensive freedom to propose aesthetically pleasing, and at the same time efficient, cooling solutions. The design is monolithic and includes the heat sink implemented as a ring with several straight fins along the body. The implementation targets a vertical orientation of the bulb, where the fins become parallel to the gravity vector and accelerate the air through a chimney effect. On the other hand, LEDs are strictly directional light sources, requiring different orientations for delivering light where needed. Thus, for positions different from vertical, the heat sink will perform inefficiently $[6,7,8]$. The presented experimental results confirm this effect as well.

Most research works investigate different variants of pin- or straight-fin heat sinks $[9,10,11,12,13]$ due to their easy parametrization and simplified geometry.
Various combinations of these primitives are often utilized for improving performance and building more complex products realizing their designer's vision. Figures 2,b) and 3,a) show expert industrial design solutions for larger industrial LEDs, designed by a project partner $^{3}$. The intricate lattice-like sinks conduct the heat away from their base and transfer it to the surrounding air ensuring redundancy of conduction and flow pathways with the aim of achieving orientation insensitive performance. The complex topologies are realized in an aluminum alloy using metal 3D printing and provide the starting point for this study. Their performance is compared numerically and experimentally to the topologically-optimized designs.

\section{Topology optimization}

Topology optimization $[14,15]$ is an iterative process, which distributes a material in a prescribed design domain by optimizing a selected performance measure; i.e. in the presented examples minimizing the average temperature of the sink base plate $[16,5]$. The result of the optimization is a voxel-based spatial distribution of zeros representing void regions and ones representing solid regions. This binary optimization problem is relaxed to a continuous density distribution in turn allowing for the use of efficient, gradient-based optimization techniques. A repetitive sequence of numerical simulations, regularization steps and design updates by mathematical programming complete the process. The topology optimization technology is now extensively utilized in the automotive and aerospace industries for solid mechanics applications, reducing weight and improving stiffness of mechanical parts with reported savings from $20 \%$ to $40 \%$. The methodology has been extended to extreme material design [17], optimized nanophotonic and acoustic devices [18, 19], electromagnetic antennas [20], and recently to thermoelectric and heat thermal energy storage devices [21, 22].

The optimization problem here is formulated as

$$
\begin{aligned}
\min _{\rho} & : J(\rho, \mathbf{u}) \\
\text { s.t. } & : r(\rho, \mathbf{u})=0, \quad \mathbf{u} \in \mathcal{U}_{\mathrm{ad}} \\
& g(\rho) \leq 0 \\
& \rho \in \mathcal{D}_{\mathrm{ad}}
\end{aligned}
$$

where the objective functional $J(\cdot)$ depends on the system response $\mathbf{u}$ and the material distribution $\rho$. The socalled density field $\rho$ takes value one in the fluid part

\footnotetext{
${ }^{3}$ AT Lighting ApS
} 


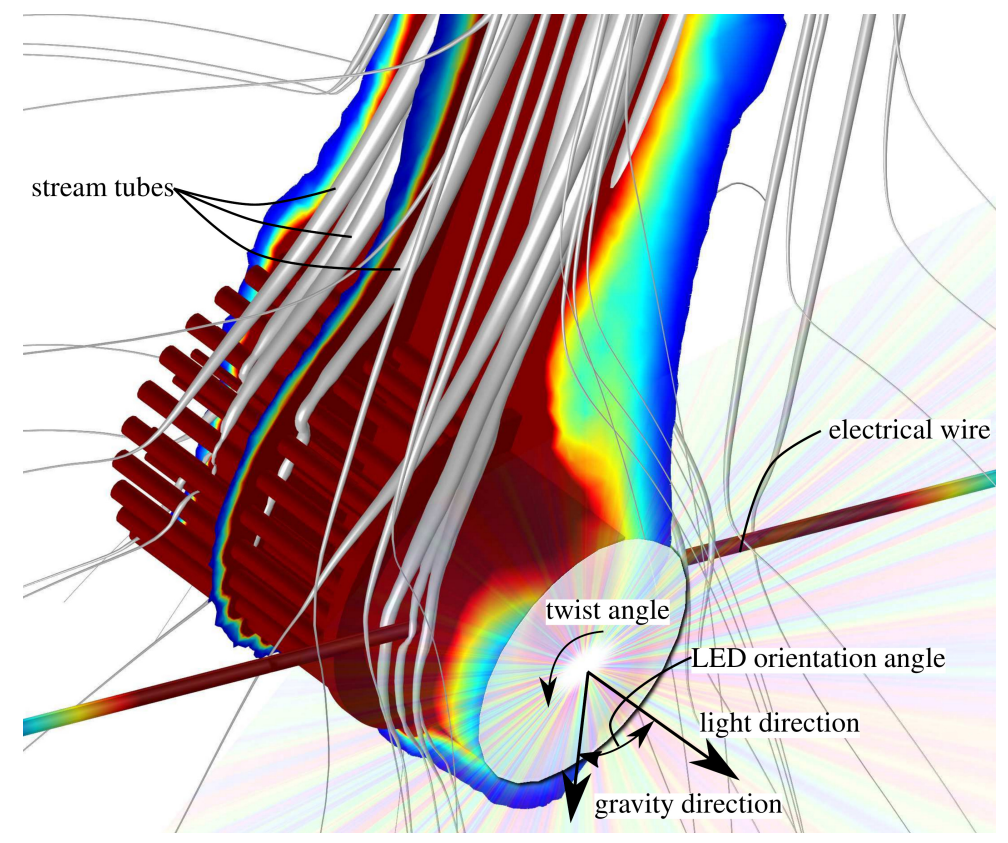

Figure 1: Example of traditional pin-fin passive LED heat sink solution. Due to the directionality of the light source robust cooling performance is required for different orientation and twist angles. Colors represent the temperature, varying from dark blue $\left(23.5^{\circ} \mathrm{C}\right)$ to dark red $\left(29.7^{\circ} \mathrm{C}\right)$.

a)

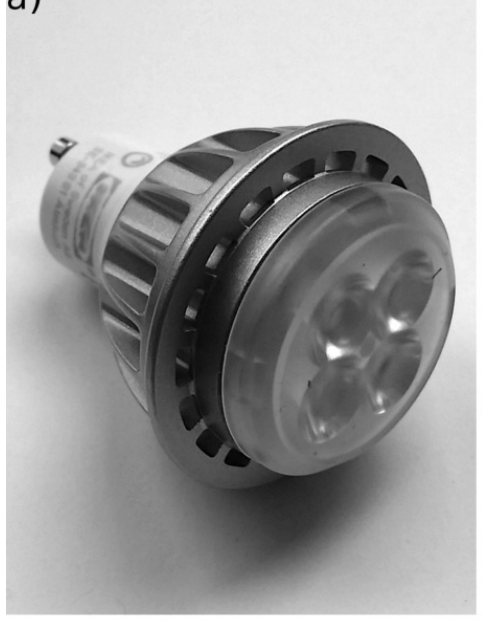

b)

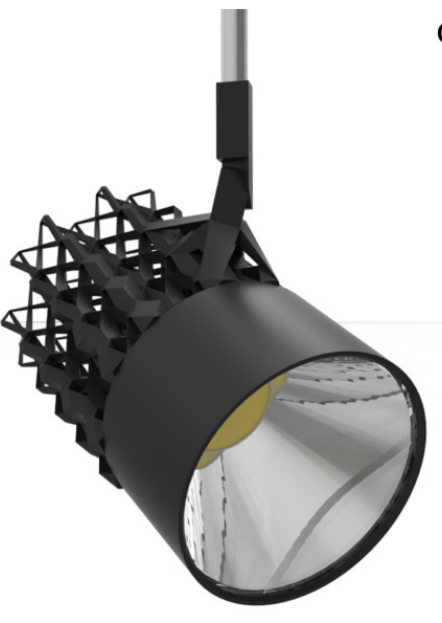

c)

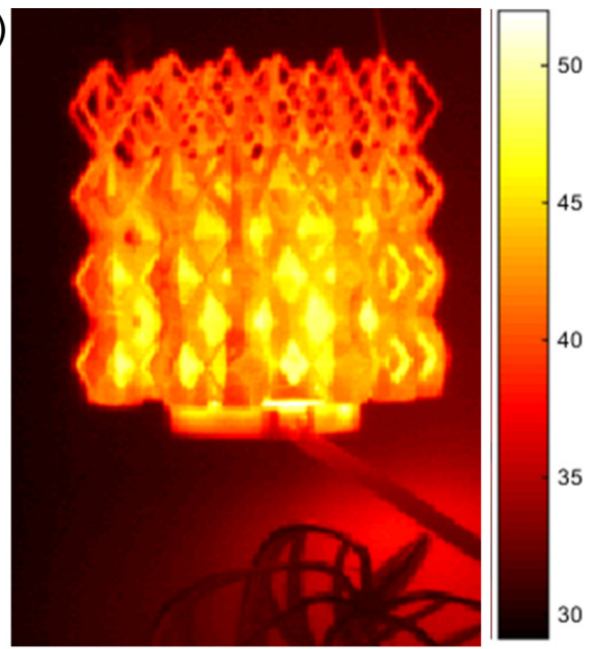

Figure 2: Examples of LED cooling solutions: a) Commercial LED bulb utilized as a direct replacement for a standard incandescent one; b) Design concept of monolithic LED lamp unit including the heat sink; c) Infrared picture of a LED unit mounted on a 3D printed heat sink. 
a)

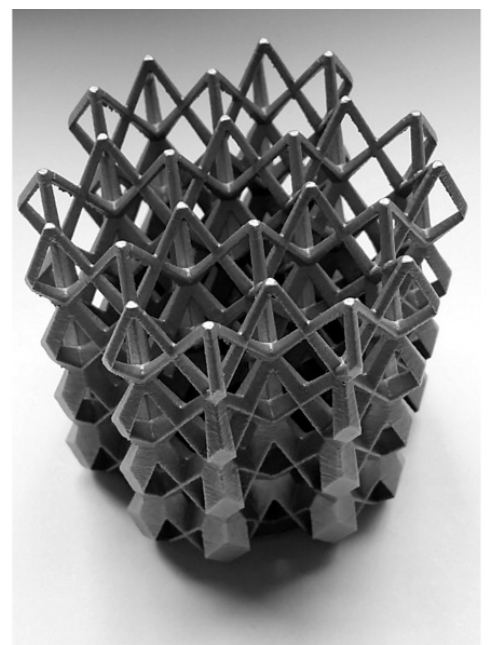

b)

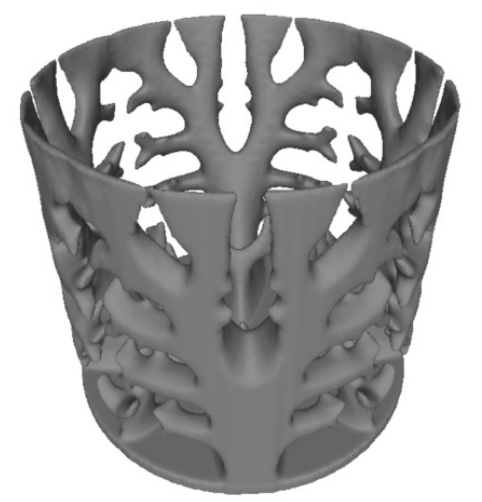

c)

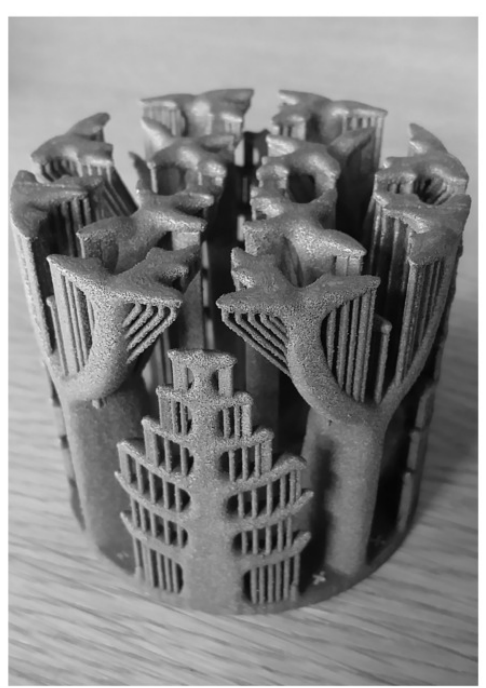

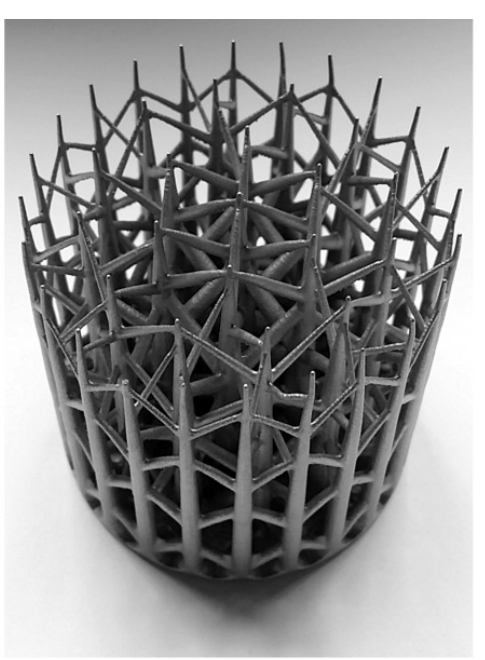
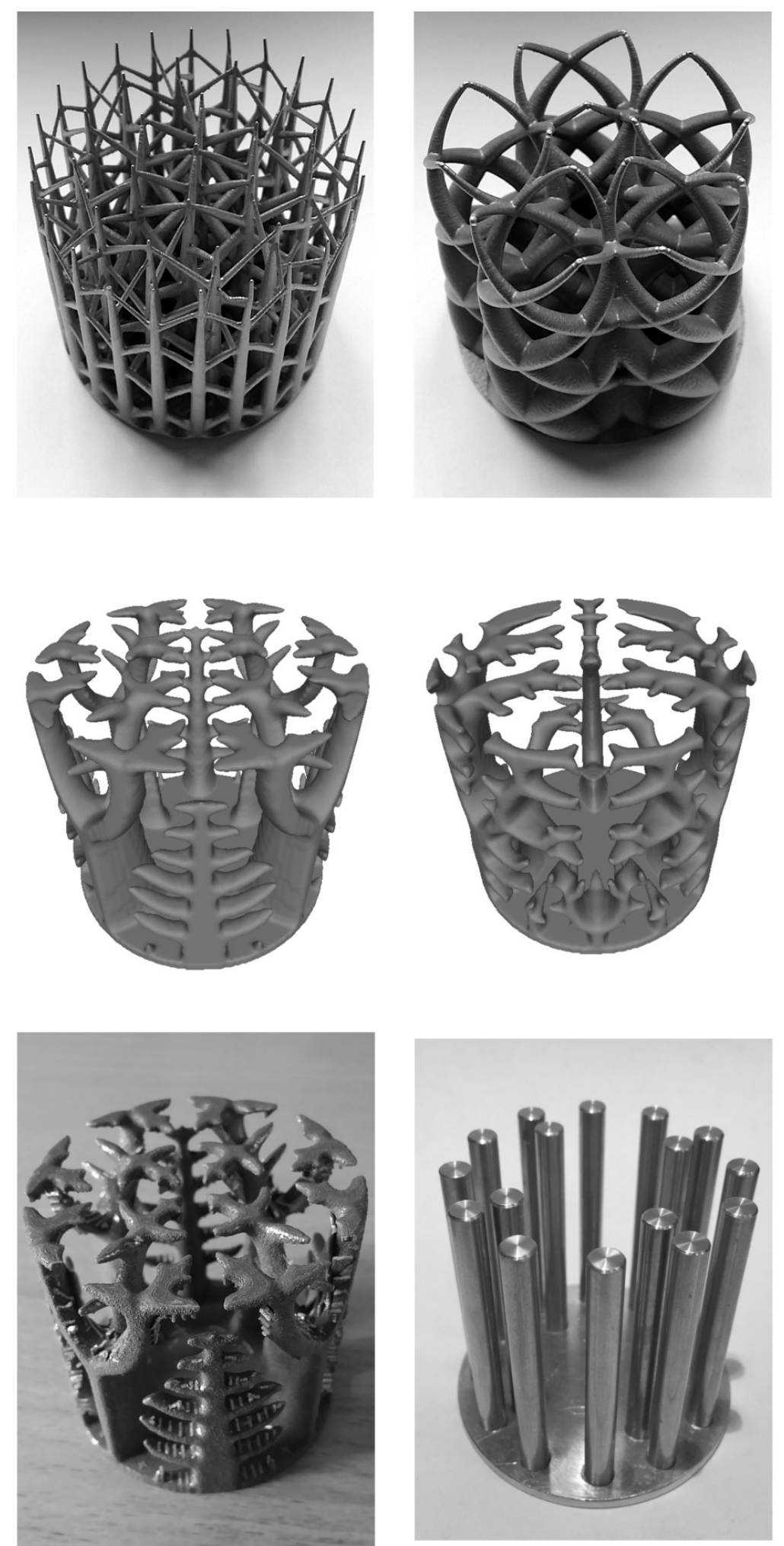

Figure 3: Heat sinks for LED light sources: a) Industrial design solutions; b) Topology-optimized LED heat sinks for vertical, and horizontal orientations with $1 / 2$ and $1 / 8$ symmetries; c) 3D printed topology-optimized design with additional support; post-processed design with removed support; and simplified interpretation manufactured by traditional techniques. 
of the computational domain and zero in the solid. The considered constraint $g(\cdot)$ imposes upper bound on the volume of the conductive material. The density field $\rho$ is required to belong to the set of admissible solutions $\mathcal{D}_{\text {ad }}$ to ensure an existence of a solution to the optimization problem. This is achieved by filtering [23].

The physics is represented by the residual $r(\rho, \mathbf{u})$ of the Navier-Stokes equations combined with a convection-diffusion equation. The response vector $\mathbf{u}=$ $\left[u_{x}, u_{y}, u_{z}, p, t\right]^{\top}$ consists of the fluid velocities $v_{(\cdot)}$ in $\mathrm{x}, \mathrm{y}$, and $\mathrm{z}$ directions, and $p$ and $t$ representing the pressure and the temperature distributions. The optimization utilizes a steady laminar incompressible fluid model coupled with heat transfer through the advection-diffusion equation. The formulation models the natural convection effect with the help of the Boussinesq approximation with details provided in $[16,5]$.

The partial differential equations are discretized using the Finite Element Method (FEM) on a regular grid of size $384 \times 384 \times 640$ (half domain) and $192 \times 192 \times 640$ (quarter domain) for the horizontal and the vertical optimization case, respectively. The fine resolution is necessary for allowing small features to appear in the optimized design. The material distribution (the density field) is represented as a constant within each element, and the discrete values are updated iteratively using gradient-based optimization techniques, i.e., a parallel version of the Method of Moving Asymptotes [24, 25]. A detailed description of the discretization and the optimization process can be found in $[5,26]$. Thus, further implementation details are omitted.

Topology optimization was first applied to fluid mechanics problems more than a decade ago [27], and only recently extended to fully-coupled conjugate heat transfer problems $[16,5]$, which provide the computational basis for the obtained results. The voxel representation provides ultimate freedom for the design process. However, finer discretizations necessary for better topological representation lead to large discrete numerical models imposing severe requirements to the computational power. Thus, applications to full assemblies, with gigavoxel resolution requirements, like an airplane wing, have only recently been demonstrated with the help of supercomputers [28]. High resolution is also required to resolve the topology and the physics in the natural convection case. The nonlinear nature of the fullycoupled Navier-Stokes and heat transfer equations further challenge the computational cost. The presented topologically-optimized designs require two to five full days of computing time running on 1000-2500 cores on a high-performance computing (HPC) cluster equipped with Intel e5-2680v2 Ivy Bridge processors. This significant computational cost is, however, surpassed by the potential savings in material and life-time costs.

Compared to parametric optimizations, regularly utilized for improving heat sink performance [9, 10], topology optimization possesses the ultimate freedom in distributing material, in turn resulting in organic-like topologies sharing similarities with natural plants and organisms. In contrast to natural evolution requiring millions of years, however, the use of efficient gradientbased solution methods speed up the generative process resulting in highly efficient solutions within hours or days. The resulting organic shapes may provide challenges for traditional subtractive manufacturing technologies or casting technologies. However, such issues may be resolved using the freedom provided by additive manufacturing, where the design is built layer-by-layer by either deposition or melting of material powder at specified points [29]. The present main restriction being requirement for self-support, i.e., upper parts should be sufficiently supported by lower parts [30,31]. As this constraint was not enforced during the present optimization, the obtained designs require additional supporting structure which must be removed manually in a postprocessing step. Recent research advances [32, 33] may provide the necessary algorithmic modifications which, in combination with a requirement for robustness to exploitation and manufacturing uncertainties, will allow for future complete digitalization of the design process $[17,23]$, thus potentially removing the need for any human intervention. However, any restriction on the design freedom will come at the cost of performance loss. Hence, in this paper the ultimate cooling capability is pursued with no additional restrictions imposed.

\section{Experimental setup}

The performance of the coolers is verified experimentally using the setup shown in Figure 4. A heat sink is positioned in the space with the help of thin threads to avoid flow disturbance. Heat is supplied by a resistor encased in a SikaBoom S isolation foam and connected to a digitally controllable Tenma 72-10480 power supply. A thermocouple 5TC-TT-KI-30-1M manufactured by OMEGA Engineering is positioned between the power source and the attached sink. A second thermocouple measures the ambient temperature near the setup. Additional digital thermometer offers independent measurements. Both thermocouples are attached to an isothermal terminal block NI TB-9214 with measurement accuracy up to $0.45^{\circ} \mathrm{C}$ and measurement sensitivity up to $0.02^{\circ} \mathrm{C}$. Data control, timing, synchronization, 
and transfer to a computer are provided by cDAQ-9171. A thin layer of Dow Corning 340 heat sink compound is utilized to prevent air pockets and to maximize heat transfer between the heat source and the attached sink.

Each measurement is obtained by first setting the supplied power and recording the temperature, with a sampling frequency of $1 \mathrm{~Hz}$, for 5400 s. All systems reached steady state in less than an hour, and the first 3600 samples are excluded from the data analysis. The rest of the record is visually inspected for abnormal variations, e.g. in ambient temperature. Only 600 sequential samples, corresponding to $10 \mathrm{~min}$ of measurement time, are extracted, and the average temperature is reported in Table 1 and Figure 7. The estimated standard deviation for all measurements is less than $0.1^{\circ} \mathrm{C}$. All measurements were obtained for ambient temperatures between $22.5^{\circ} \mathrm{C}$ and $24.3^{\circ} \mathrm{C}$.

\section{Design parameters, assumptions and simplifica- tions}

For experimental and simulation purposes the LED element is replaced by a heat-generating element attached to a $3 \mathrm{~mm}$ thick aluminum plate and encased in foam to direct the heat towards the sink. Due to the high computational cost necessary for obtaining optimized topologies, designs are generated for a controllable power source of $1.08 \mathrm{~W}$, and later their performances are numerically and experimentally tested for input powers up to $5.27 \mathrm{~W}$. The Grashof number, which is lower than $7.3 \times 10^{5}$ based on the heat sink diameter, is smaller than the transition limit for horizontal cylinders, and hence, verifies the steady laminar flow assumption. Two different orientations are considered, horizontal (Figure 5) and vertical (Figure 6), with designs shown in Figure 3. The horizontal designs are optimized with $1 / 2$ and $1 / 8$ symmetries and the vertical with $1 / 8$ symmetry. Increased symmetry means reduced sensitivity to rotation. The optimized topology for horizontal orientation with $1 / 8$ symmetry is manufactured and tested against several custom designed, off-the-shelf and simplified pin-fin designs with results shown in Table 1 .

For the considered optimization case a prescribed volume of aluminum is distributed in a design domain occupied by air. The design domain is embedded in a simulation domain which has the form of a rectangular parallelepiped with a square base and dimensions $150 \mathrm{~mm} \times 150 \mathrm{~mm} \times 250 \mathrm{~mm}$. The design domain is a cylinder with diameter of $65 \mathrm{~mm}$ and height of $60 \mathrm{~mm}$. The central axis of the cylinder is either parallel to (vertical) or perpendicular (horizontal) to the gravity direc- tion. The design domain is connected to a heat source element encased in a foam insulation to direct the heat towards the heat sink. The heat is generated by a resistor which is attached to a $5 \mathrm{~mm}$ thick round aluminum plate, with diameter $52 \mathrm{~mm}$, in order to distribute the heat supplied to the design domain more evenly. The total thickness of the foam encasement is $25 \mathrm{~mm}$.

During the optimization, the whole setup, which consist of a design domain and the attached heat source part, is positioned centrally in the simulation domain. The conductivity of the aluminum alloy distributed in the design domain is $k=175 \mathrm{~W} \cdot \mathrm{m}^{-1} \cdot \mathrm{K}^{-1}$ and the heat capacity is $c_{p}=903 \mathrm{~J} \cdot \mathrm{kg}^{-1} \cdot \mathrm{K}^{-1}$, width density $\rho=2702 \mathrm{~kg} \cdot \mathrm{m}^{-3}$. The considered physical properties of the air are $k=0.026 \mathrm{~W} \cdot \mathrm{m}^{-1} \cdot \mathrm{K}^{-1}, c_{p}=1003 \mathrm{~J} \cdot \mathrm{kg}^{-1} \cdot \mathrm{K}^{-1}$, and $\rho=1.2 \mathrm{~kg} \cdot \mathrm{m}^{-3}$. The dynamic viscosity of air is $\mu=1.86 \times 10^{-5} \mathrm{~Pa} \cdot \mathrm{s}$ and the coefficient of volumetric expansion is $\beta=3.34 \times 10^{-3} \mathrm{~K}^{-1}$. The insulation is manufactured using SikaBoom $\mathrm{S}$ foam with properties $k=0.026 \mathrm{~W} \cdot \mathrm{m}^{-1} \cdot \mathrm{K}^{-1}, c_{p}=1003 \mathrm{~J} \cdot \mathrm{kg}^{-1} \cdot \mathrm{K}^{-1}$. The copper wires connecting the resistor to the power supply are considered in the simulation as well. Their thickness is $3 \mathrm{~mm}$ with thermal properties $k=287 \mathrm{Wm}^{-1} \cdot \mathrm{K}^{-1}$, $c_{p}=376 \mathrm{~J} \cdot \mathrm{kg}^{-1} \cdot \mathrm{K}^{-1}$, and $\rho=8800 \mathrm{~kg} \cdot \mathrm{m}^{-3}$.

To lower the manufacturing cost, a simplified traditional pin-fin heat sink, as shown in Figure 3, is constructed based on the optimized results. The base plate dimensions of the pin-fin cooler are kept the same as the topology optimized one. The rest of material is distributed in two circular layers of aluminum rods mimicking the optimized material distribution. The diameter of the rods is $5.98 \mathrm{~mm}$. The outer layer with a diameter of $53 \mathrm{~mm}$ consists of 12 rods and the inner layer with a diameter of $37 \mathrm{~mm}$ consists of 4 rods.

The verifications are performed using COMSOL Multiphysics ${ }^{4}$ with extended simulation domain $300 \mathrm{~mm} \times 300 \mathrm{~mm} \times 1050 \mathrm{~mm}$ in order to possibly match open space conditions, i.e., to decrease the effect of the boundary conditions on the design response. The axis of the heat sink setup is positioned $125 \mathrm{~mm}$ from the bottom of the extended verification domain. All outer boundaries have open flow boundary conditions with an exception applied on the bottom where zero fluid velocity is enforced. For the temperature field all boundaries are set to an ambient air temperature of $23.5^{\circ} \mathrm{C}$ with an exception applied on the upped side where a zero diffusive flux is enforced.

${ }^{4}$ Finite element analysis, solver and multiphysics simulation software. 

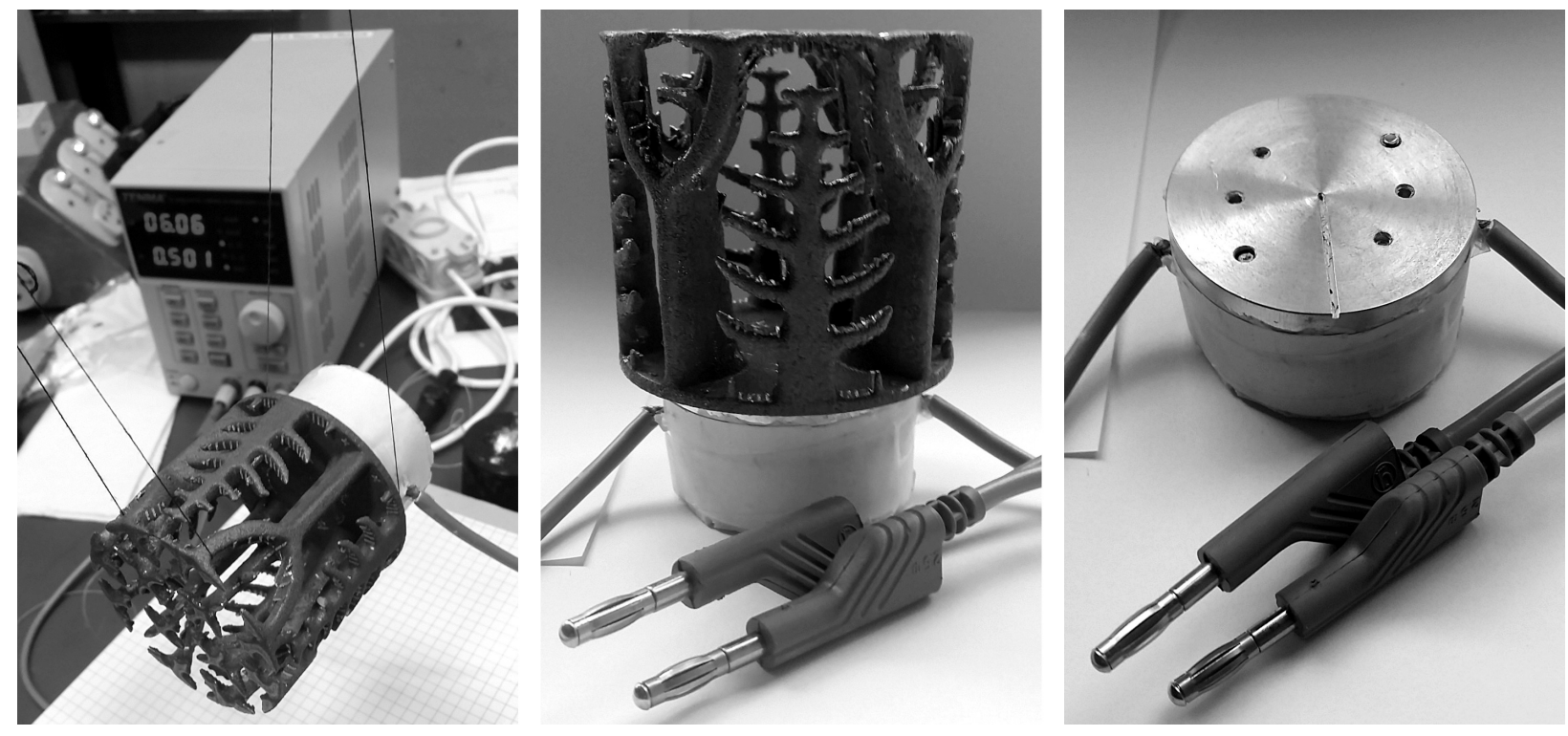

Figure 4: Setup for experimental verification of a heat sink (3D printed heat sink in horizontal position, a heat sink attached to a heat source, a heat source element without heat sink). Each heat sink is oriented and positioned with the help of thin threads. During the experiment, any of the sinks is attached to a heat source. The heat source element consists of a resistor encased in an insulation foam and connected with two copper wires to a power source. The thin channel is utilized to position a thermocouple between the heat sink and the heat distribution plate.

\section{Results and discussions}

Detailed numerical simulations for horizontal orientation are performed for three of the designs which are compared to experimental ones in Figure 7. The results for $1.08 \mathrm{~W}$ power input are shown in Figure 5. The computational results agree well with the experimentally measured temperature at the center of the base sink plate, thus, providing confidence in the simulations and a foundation for a deeper investigation of the heat transfer mechanism by using the easy access to the numerically evaluated additional physical fields such as flow velocity, pressure, and temperature distribution.

The temperature gradients for all three cases accelerates the air to pass through and to extract heat from the sinks. The flow velocities are higher for the topologyoptimized and pin designs resulting in increased massflow and convective effects. The air is redirected around the lattice design in contrast to the others, where the flow passes through and accelerates in a low-pressure cavity in the middle of the models. This effect leads to an almost uniform temperature distribution in the lattice sink. Cold air is sucked in and heated to the sink temperature through the lowest elements. The hot air cannot extract additional heat from the upper part, thus, making it redundant. On the other hand, horizontal and vertical temperature variations are observed for both the optimized and the interpreted designs indicating more efficient heat transport both through conduction in the sink and through convection to the moving fluid. Thus, the optimized design achieves $21 \%-23 \%$ lower temperature for $17 \%$ smaller amount of material. Interpretation and post-processing of the results move the performance away from the optimal point, however, compared to the original lattice design the temperature is $4 \%$ to $6 \%$ lower for even higher $19 \%$ savings of material. It is interesting to note that the surface areas of the optimized and pin designs, $2.36 \times 10^{-2} \mathrm{~m}^{2}$ and $2.10 \times 10^{-2} \mathrm{~m}^{2}$ respectively, are significantly lower than the $2.55 \times 10^{-2}$ $\mathrm{m}^{2}$ for the lattice sink. This fact demonstrates that better performance cannot be achieved just by maximizing the area or the amount of material, but requires delicate balancing between the heat conducted through the material, the temperature gradient necessary for accelerating the flow, the velocity pattern and the contact area between the solid and the fluid. This complex problem, difficult to apprehend even by trained engineers and designers, is captured by the topology optimization, resulting in high performing and at the same time economical optimized solutions.

The above conclusion is also supported by the experimental results in Table 1, where the commercial offthe-shelf cooler (column 5) performs worse in horizontal orientation than the topology-optimized one (column 2 ). Changing the orientation to vertical gives an advantage to the commercial sink allowing it to accelerate 

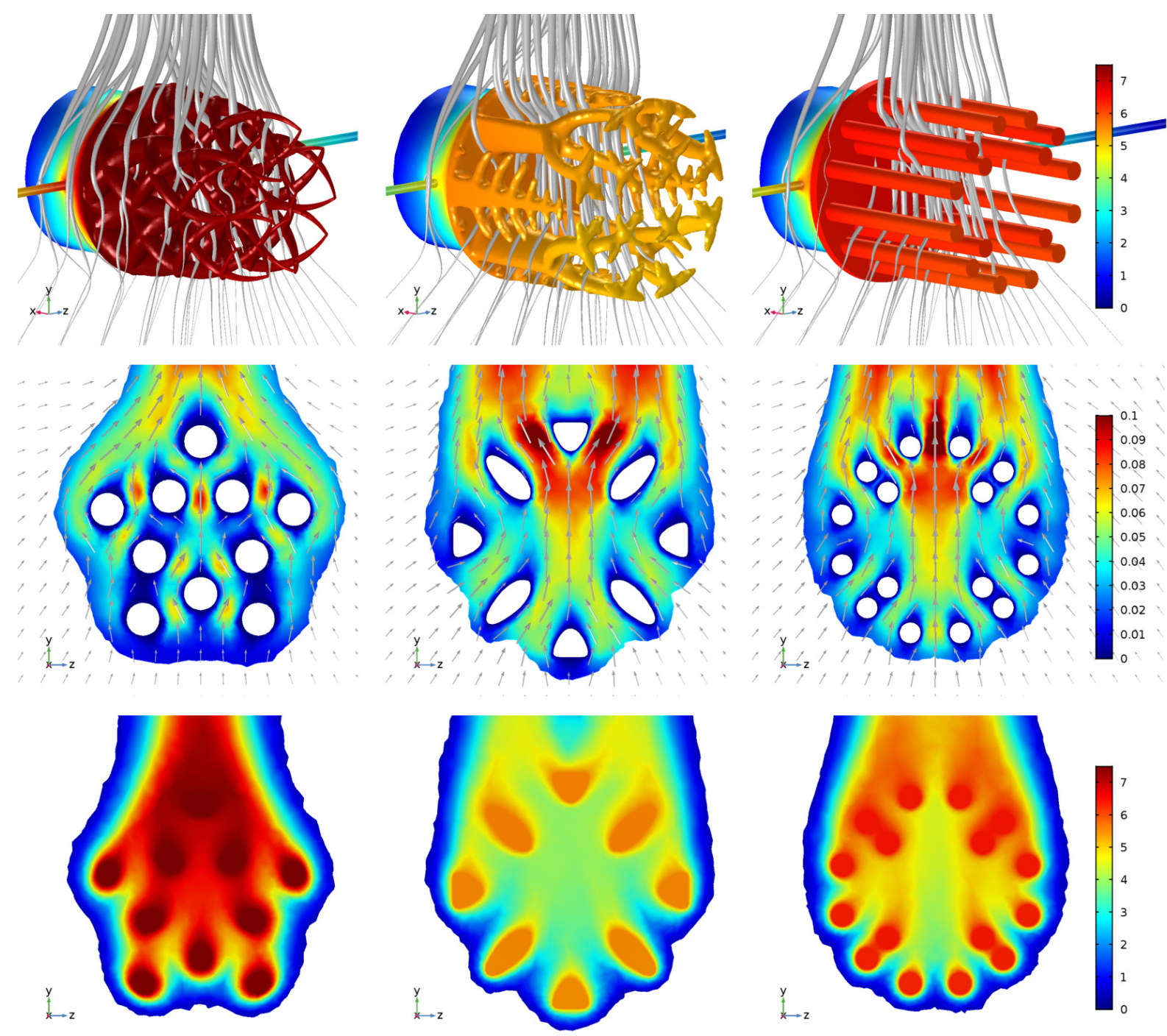

Figure 5: Numerical modeling of natural convection around horizontal reference heat sink, topology-optimized heat sink, and a simplified heat sink, obtained by post-interpretation of the topological design and manufactured by traditional techniques. The first row presents the temperature difference to ambient temperature $\left[{ }^{\circ} \mathrm{C}\right]$ in the sinks with stream tubes. The second row shows the velocity $[\mathrm{m} / \mathrm{s}]$ of the cooling air, and the third row provides temperature $\left[{ }^{\circ} \mathrm{C}\right]$ distribution in a vertical $2 \mathrm{D}$ cross-section of the modeling region perpendicular to the sinks axes. The cross-section is located in the middle of the sink. 

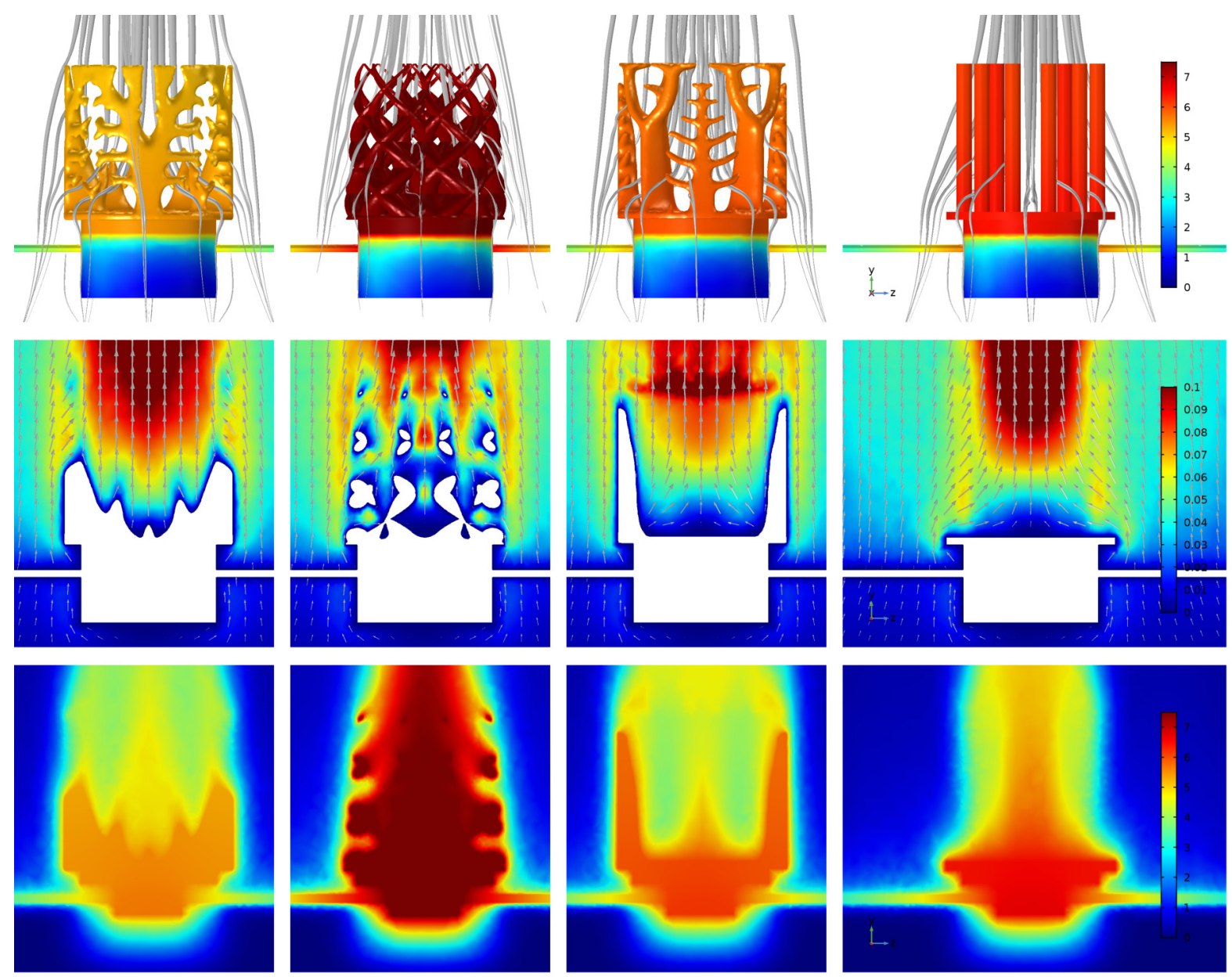

Figure 6: Numerical modeling of natural convection around topology-optimized heat sink for vertical orientation, reference heat sink, topologyoptimized heat sink for horizontal orientation in a vertical position and a simplified heat sink. The first row presents the temperature difference to ambient temperature $\left[{ }^{\circ} \mathrm{C}\right]$ in the sinks with stream tubes. The second row shows the velocity $[\mathrm{m} / \mathrm{s}]$ of the cooling air, and the third row provides temperature $\left[{ }^{\circ} \mathrm{C}\right]$ distribution in a vertical $2 \mathrm{D}$ cross-section of the modeling region parallel to the sinks axes. 
a)

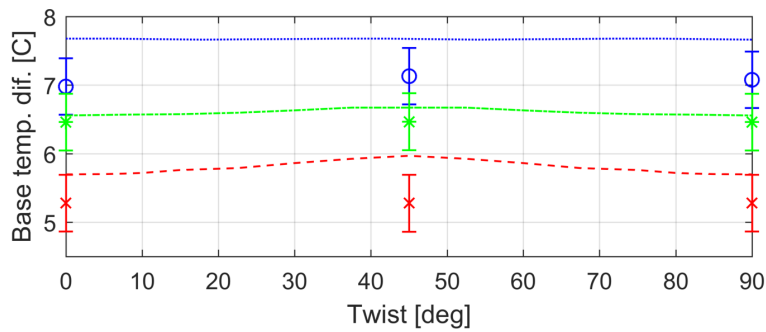

b)

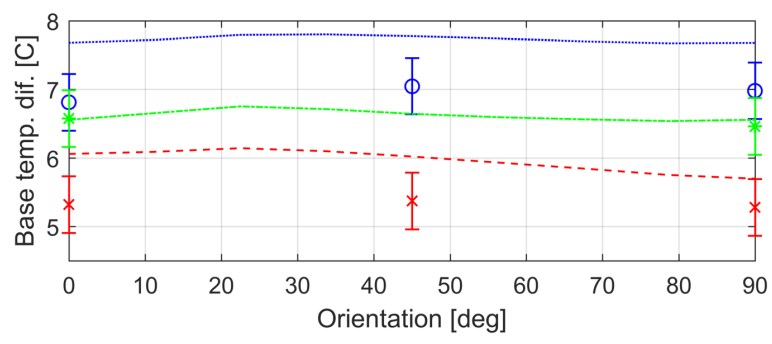

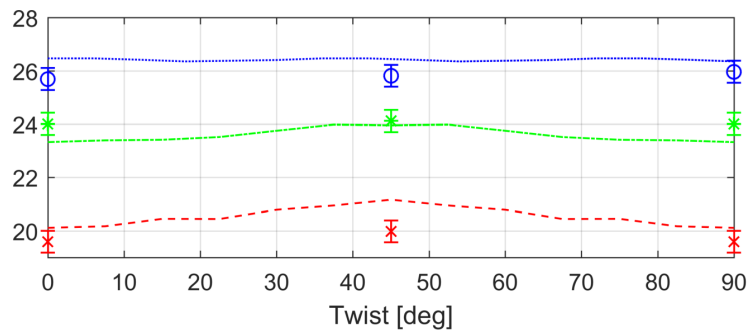

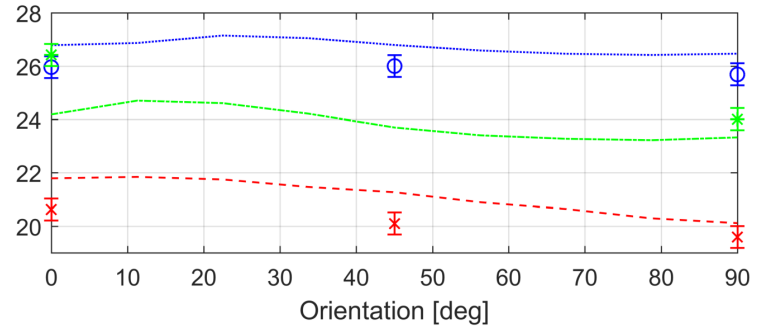

c)

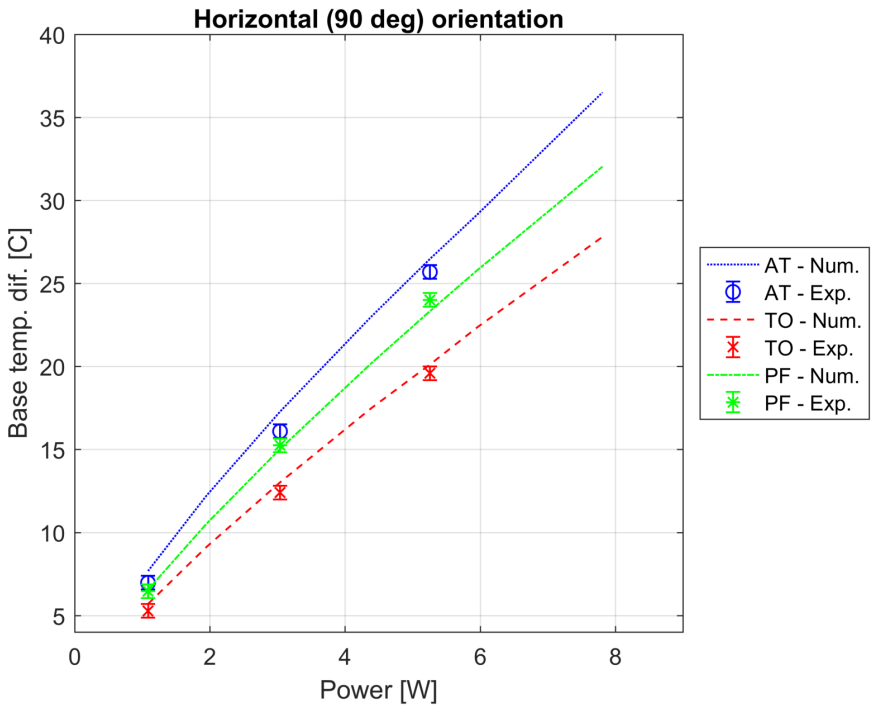

Figure 7: Comparison of numerically simulated and experimentally measured base temperature difference for reference heat sink (AT), topologyoptimized heat sink (TO), and simplified heat sink (PF) shown in Figure 5. Subfigures a) and b) show trends for 1.08W and 5.27W power input and different orientation and twist angles. Subfigure c) demonstrates results for input powers from $1 \mathrm{~W}$ to $8 \mathrm{~W}$. 


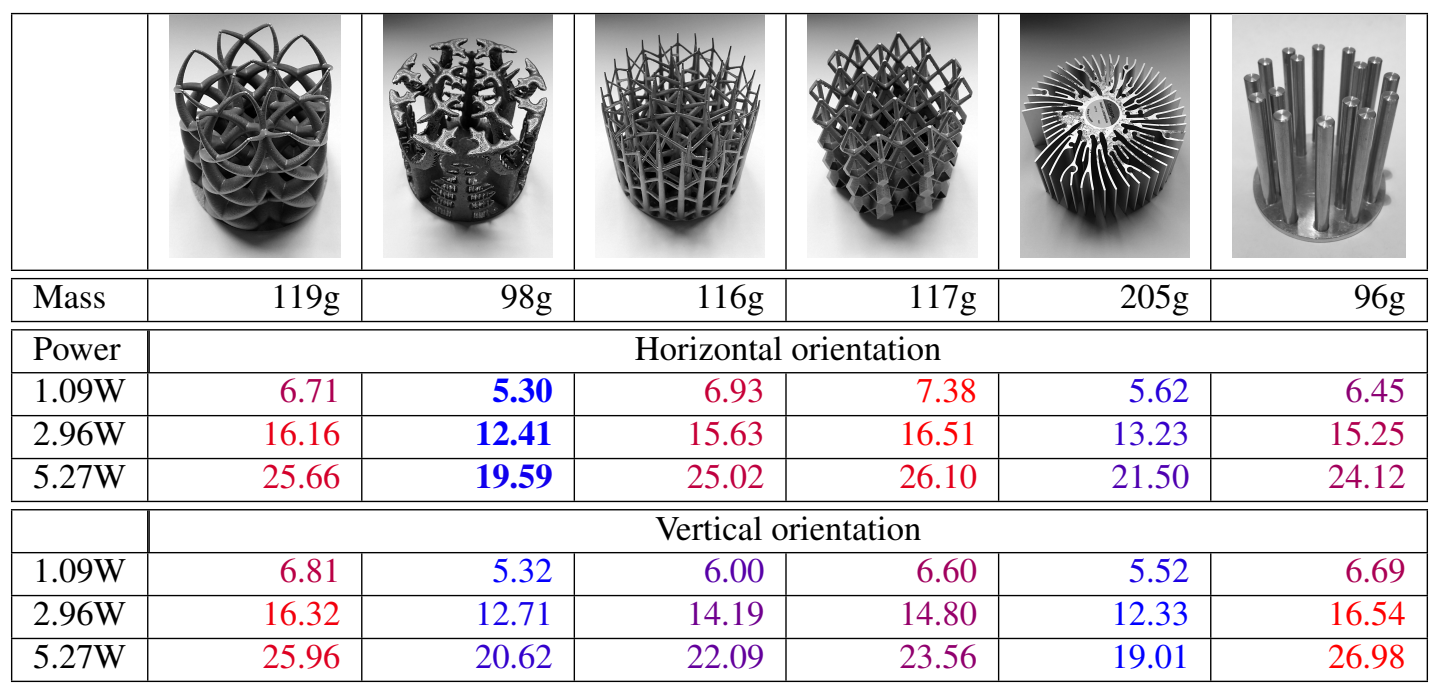

Table 1: The table presents experimental measurements of the difference between the temperature at the center of the heat sink plate and the ambient temperature for different power inputs, and two orientations. Text coloring between blue (cold) and red (hot) is scaled according to the devices with maximum and minimum performance for each driving power. The topology optimized design (second column) outperforms all other designs for an experimental setup corresponding to the optimization settings, i.e., horizontal orientation. In a vertical position, the optimized design is outperformed only by the twice heavier and much bigger commercial sink in column 5 .

the air between the plates and using the large surface to transfer the heat to the flow. For low power input, the topology-optimized design outperforms it slightly indicating that the temperature gradient cannot accelerate the flow enough. It is interesting to note that the sink optimized for horizontal orientation performs better than any of the lattice designs in a vertical test position. The explanation is revealed by the numerical experiments shown in Figure 6. All coolers suck in cold air close to the base plate, however, similar to the horizontal case, the lattice structure prevents the air from accelerating and passing through, thus, heating the air at the bottom, in turn making the upper part of the sink redundant. The topology-optimized sinks use the inner chamber to accelerate the flow and to create a stronger chimney effect. The bent parts at the top of the optimized design for horizontal position increase the drag, resulting in lower flow velocity. The other important difference between the design optimized for horizontal position, the post-processed one, and the one for vertical orientation is the velocity pattern close to the base plate. The latter provides gradually decreasing material distribution towards the center and higher flow velocities capable of extracting heat from the base plate delivered by the solid due to its higher conductivity. A more detailed analysis, based on numerical simulations, of the design features can be found in [26].

As expected, the lowest base temperature is obtained for the orientation corresponding to the actual optimization conditions. The loss of performance for the horizontally optimized cooler is less than $5 \%$ for positions varying from 0 to 90 degrees. An even flatter curve can potentially be realized by optimizing simultaneously for all desired orientations, however, due to the high computational demands, such a study is left for future investigations. The imposed $1 / 8$ symmetry provides negligible sensitivity to rotations around the sink axis demonstrating a computationally cheaper alternative for achieving orientational independence.

\subsection{Lifetime expectancy}

As the manufacturing processes of LEDs and microelectronic products share many similarities, the methodologies for estimating the lifetime of microelectronic components are applicable for reliability analysis of light-emitting diode products [34]. A $10^{\circ} \mathrm{C}$ decrease of the operating temperature doubles the life of a product in the semiconductor industry. This rough estimate is based on the empirical Arrhenius model for describing reaction kinetics in chemistry and physics.

$$
k=A \mathrm{e}^{-\frac{E_{A}}{k_{B} t}}
$$

The model is often utilized to represent the damage $k$ of LEDs over time, and depends on the activation energy $E_{A}$ associated with the reaction and estimated from experiments, the Boltzmann constant $\left(k_{B}=\right.$ 
$\left.8.617 \times 10^{-5} \mathrm{eV} / \mathrm{K}\right)$ and the absolute temperature $t$. For activation energies varying from $E_{A}=0.4 \mathrm{eV} / \mathrm{K}$ to $E_{A}=0.6 \mathrm{eV} / \mathrm{K}[35]$ and horizontal orientation with input power of $5.27 \mathrm{~W}$, the estimated increase of the lifetime for the presented topology-optimized heat sink is from $40 \%$ to $60 \%$. Increasing the input power to $8 \mathrm{~W}$, in general, shortens the life expectancy, and in this case, the utilization of the topology-optimized heat sink doubles the lifetime of the LED. It should be pointed out, that it is unlikely for LEDs to fail completely. Usually, their light intensity will deteriorate over time. Thus the estimated increase of reliability is directly reflected in the maintenance cost, i.e., $50 \%$ increase of lifetime is directly proportional to $50 \%$ decrease in maintenance cost of new LED installations, in addition to the existing ones, during the utilization period.

\section{Conclusions}

Our holistic topology optimization approach results in significant material savings and design improvements of passive LED coolers. The possibility to account for several physical fields and their complex interaction provides close to optimal utilization of the heat sink material and moves performance bounds close to the limit. For such broadly applied and well-studied devices, reduction of $17 \%$ in material utilization in unison with a $21-23 \%$ temperature decrease is still achievable, fully demonstrating the potential of the presented approach. The topology-optimized designs can be produced using $3 \mathrm{D}$ printing techniques or can be simplified further making them suitable for traditional manufacturing with a small loss of performance. Higher computational effort is required to account for additional physical effects and practical constraints. The benefits in material savings and decreased temperature justify this cost increase in the design phase. Extensions to more complex multiphysical interactions including heat transfer in turbulent flows, magnetic refrigeration or elastocaloric cooling are still in their infancy, but cater for further future improvements in energy consumption of thermal and other systems.

[1] Y. Chan, R. Kantamaneni, Study on energy efficiency and energy saving potential in industry and on possible policy mechanisms, Tech. rep., ICF Consulting Ltd under contract to European Commission Directorate-General Energy (2015).

[2] Navigant Consulting, Inc., Energy savings forecast of solid-state lighting in general illumination applications, Tech. rep., U.S. Department of Energy (2016).

[3] E. M. Dede, S. N. Joshi, F. Zhou, Topology optimization, additive layer manufacturing, and experimental testing of an aircooled heat sink, Journal of Mechanical Design 137 (11) (2015) 111403-111403-9.
[4] P. Coffin, K. Maute, Level set topology optimization of cooling and heating devices using a simplified convection model, Structural and Multidisciplinary Optimization 53 (5) (2015) 9851003.

[5] J. Alexandersen, O. Sigmund, N. Aage, Large scale threedimensional topology optimisation of heat sinks cooled by natural convection, International Journal of Heat and Mass Transfer 100 (2016) 876 - 891.

[6] D. Jang, S.-J. Park, S.-J. Yook, K.-S. Lee, The orientation effect for cylindrical heat sinks with application to led light bulbs, International Journal of Heat and Mass Transfer 71 (2014) 496 502.

[7] Q. Shen, D. Sun, Y. Xu, T. Jin, X. Zhao, Orientation effects on natural convection heat dissipation of rectangular fin heat sinks mounted on leds, International Journal of Heat and Mass Transfer 75 (2014) 462 - 469.

[8] B. Li, Y.-J. Baik, C. Byon, Enhanced natural convection heat transfer of a chimney-based radial heat sink, Energy Conversion and Management 108 (2016) $422-428$.

[9] S.-H. Yu, K.-S. Lee, S.-J. Yook, Optimum design of a radial heat sink under natural convection, International Journal of Heat and Mass Transfer 54 (11) (2011) 2499 - 2505.

[10] D. Jang, S.-H. Yu, K.-S. Lee, Multidisciplinary optimization of a pin-fin radial heat sink for $\{$ LED $\}$ lighting applications, International Journal of Heat and Mass Transfer 55 (4) (2012) 515 521.

[11] D. Jang, S.-J. Yook, K.-S. Lee, Optimum design of a radial heat sink with a fin-height profile for high-power led lighting applications, Applied Energy 116 (2014) 260 - 268.

[12] V. A. Costa, A. M. Lopes, Improved radial heat sink for led lamp cooling, Applied Thermal Engineering 70 (1) (2014) $131-138$.

[13] S.-J. Park, D. Jang, S.-J. Yook, K.-S. Lee, Optimization of a chimney design for cooling efficiency of a radial heat sink in a led downlight, Energy Conversion and Management 114 (2016) $180-187$.

[14] M. P. Bendsøe, N. Kikuchi, Generating optimal topologies in structural design using a homogenization method, Computer Methods in Applied Mechanics and Engineering 71 (2) (1988) $197-224$.

[15] M. P. Bendsøe, O. Sigmund, Topology Optimization - Theory, Methods and Applications, Springer Verlag, Berlin Heidelberg, 2003.

[16] J. Alexandersen, N. Aage, C. S. Andreasen, O. Sigmund, Topology optimisation for natural convection problems, International Journal for Numerical Methods in Fluids 76 (10) (2014) 699721. doi: $10.1002 / \mathrm{fld} .3954$.

[17] E. Andreassen, B. S. Lazarov, O. Sigmund, Design of manufacturable 3d extremal elastic microstructure, Mechanics of Materials 69 (1) (2014) $1-10$.

[18] J. Jensen, O. Sigmund, Topology optimization for nanophotonics, Laser \& Photonics Reviews 5 (2) (2011) 308-321. doi:10.1002/lpor.201000014.

[19] J. Andkjaer, O. Sigmund, Topology optimized cloak for airborne sound, Journal of Vibration and Acoustics 135 (4) (2013) 041011-041011. doi:10.1115/1.4023828.

[20] T. Nomura, K. Sato, K. Taguchi, T. Kashiwa, S. Nishiwaki, Structural topology optimization for the design of broadband dielectric resonator antennas using the finite difference time domain technique, International Journal for Numerical Methods in Engineering 71 (11) (2007) 1261-1296. doi:10.1002/nme.1974.

[21] S. Soprani, J. Haertel, B. Lazarov, O. Sigmund, K. Engelbrecht, A design approach for integrating thermoelectric devices using topology optimization, Applied Energy 176 (2016) 49 - 64. doi:http://dx.doi.org/10.1016/j.apenergy.2016.05.024.

[22] A. Pizzolato, A. Sharma, K. Maute, A. Sciacovelli, V. Verda, 
Design of effective fins for fast pcm melting and solidification in shell-and-tube latent heat thermal energy storage through topology optimization, Applied Energy 208 (Supplement C) (2017) 210 - 227. doi:https://doi.org/10.1016/j.apenergy.2017.10.050.

[23] B. S. Lazarov, F. Wang, O. Sigmund, Length scale and manufacturability in density-based topology optimization, Archive of Applied Mechanics 86 (1) (2016) 189-218. doi:10.1007/s00419-015-1106-4.

[24] K. Svanberg, The method of moving asymptotes - a new method for structural optimization, International Journal for Numerical Methods in Engineering 24 (1987) 359-373.

[25] N. Aage, E. Andreassen, B. Lazarov, Topology optimization using petsc: An easy-to-use, fully parallel, open source topology optimization framework, Structural and Multidisciplinary Optimization 51 (3) (2015) 565-572. doi:10.1007/s00158-0141157-0.

[26] J. Alexandersen, O. Sigmund, K. Meyer, B. Lazarov, Design of passive coolers for light-emitting diode lamps using topology optimisation, International Journal of Heat and Mass TransferIn press. doi:10.1016/j.ijheatmasstransfer.2018.01.103.

[27] T. Borrvall, J. Petersson, Topology optimization of fluids in stokes flow, International Journal for Numerical Methods in Fluids 41 (1) (2003) 77-107. doi:10.1002/fld.426.

[28] N. Aage, E. Andreassen, B. S. Lazarov, O. Sigmund, Gigavoxel computational morphogenesis for structural design, Nature 550 (7674) (2017) 84 - 86. doi:10.1038/nature23911.

[29] I. Gibson, D. Rosen, B. Stucker, Additive Manufacturing Technologies: 3D Printing, Rapid Prototyping, and Direct Digital Manufacturing, Springer New York, New York, NY, 2015.

[30] D. Wang, Y. Yang, Z. Yi, X. Su, Research on the fabricating quality optimization of the overhanging surface in slm process, The International Journal of Advanced Manufacturing Technology 65 (9) (2013) 1471-1484. doi:10.1007/s00170-012-4271-4.

[31] R. Mertens, S. Clijsters, K. Kempen, J.-P. Kruth, Optimization of scan strategies in selective laser melting of aluminum parts with downfacing areas, Journal of Manufacturing Science and Engineering 136 (6) (2014) 061012-061012-7. doi:10.1115/1.4028620.

[32] A. T. Gaynor, J. K. Guest, Topology optimization considering overhang constraints: Eliminating sacrificial support material in additive manufacturing through design, Structural and Multidisciplinary Optimization 54 (5) (2016) 1157-1172. doi:10.1007/s00158-016-1551-x.

[33] M. Langelaar, Topology optimization of $3 \mathrm{~d}$ selfsupporting structures for additive manufacturing, Additive Manufacturing $12 \quad$ (2016) $60 \quad-\quad 70$. doi:http://dx.doi.org/10.1016/j.addma.2016.06.010.

[34] M.-H. Chang, D. Das, P. Varde, M. Pecht, Light emitting diodes reliability review, Microelectronics Reliability 52 (5) (2012) 762 - 782. doi:https://doi.org/10.1016/j.microrel.2011.07.063.

[35] C. Qian, X. Fan, J. Fan, C. Yuan, G. Zhang, An accelerated test method of luminous flux depreciation for led luminaires and lamps, Reliability Engineering \& System Safety 147 (Supplement C) (2016) 84 - 92. doi:https://doi.org/10.1016/j.ress.2015.11.009. 